\title{
Erratum to: On the prospects of a clause combining approach to "focusing" no-constructions in Malagasy
}

\section{Hans-Martin Gärtner}

Published online: 26 March 2010

(C) Springer Science+Business Media B.V. 2010

Erratum to: Nat Lang Linguist Theory (2009) 27: 789-816

DOI 10.1007/s11049-009-9077-1

Due to a typesetting fault, the following errors were not corrected. Springer apologizes for this oversight.

- p. 796

WRONG VERSION: (18) $\operatorname{MOST}(\lambda z .|B O Y|(z))(\lambda y[y=\iota x . S T U D E N T(x)])$

CORRECT VERSION: (18) $\operatorname{MOST}(\lambda z . B O Y(z))(\lambda y[y=\iota x . \operatorname{STUDENT}(x)])$

- p. 796

WRONG VERSION: ... (18) can only be true if $B O Y \leq 1$.

CORRECT VERSION: ... (18) can only be true if $|B O Y| \leq 1$.

The online version of the original article can be found under doi:10.1007/s11049-009-9077-1.

H.-M. Gärtner $(\bowtie)$

Zentrum für Allgemeine Sprachwissenschaft (ZAS), Schützenstr. 18, 10117 Berlin (Mitte), Germany

e-mail: gaertner@zas.gwz-berlin.de 